\title{
Determinação do tamanho da amostra de papéis hidrossensíveis em experimentos ligados à tecnologia de aplicação
}

\section{Determination of the samples required of water-sensitive paper in experiments related spray technology}

\author{
Alexandre Ballarotti Nascimento ${ }^{1}$; Gustavo Migliorini de Oliveira ${ }^{2 *}$; \\ Inês Cristina de Batista Fonseca ${ }^{3}$; Otavio Jorge Grigoli Abi Saab; \\ Marcelo Giovanetti Canteri ${ }^{3}$
}

\section{Resumo}

O aumento no número de amostras coletadas aumenta o nível de confiança das estimativas. Entretanto, pouco se sabe acerca do número de cartões de papel hidrossensível que devem ser usados em trabalhos de tecnologia de aplicação. Assim, o objetivo deste trabalho foi determinar o número mínimo de cartões de papel hidrossensível a serem utilizados em experimentações, tendo como base as variáveis: porcentagem de cobertura, número de gotas por $\mathrm{cm}^{2}$ e diâmetro mediano volumétrico (DMV). Realizouse um experimento em esquema fatorial $2 \times 2$ com cinco repetições, contendo cada repetição 36 cartões de papel hidrossensível. Com o intuito de obter relativa variabilidade entre os tratamentos, os fatores envolvidos foram pontas de pulverização (TR110-01 e ULD120-015) e pressões de trabalho (138 e 276 $\mathrm{kPa})$, uma vez que o tamanho de gotas é modificado tanto pela ponta de pulverização como pelo pressão de trabalho utilizada. Como precisão das estimativas, as margens de erros assumidas foram $20 \%, 15 \%$ e $10 \%$ da média, o que gerou números de amostras de: 7,12 e 26 , para porcentagem de cobertura; 5, 8 e 16, para número de gotas por $\mathrm{cm}^{2}$; e 1,2 e 4, para diâmetro mediano volumétrico (DMV), respectivamente. Análises de variância, seguidas de testes Tukey à $5 \%$ de probabilidade foram feitas para a média dos 36 cartões de papel hidrossensível, bem como para a média do número mínimo de papéis (amostras) determinado. Comparando-se estatisticamente os resultados obtidos em cada variável para $n=36$ amostras e $n=$ amostras a $20 \%$ de erro, nota-se que os resultados são idênticos em ambas as situações, demonstrando que taxas de aplicações, bem como tamanhos de gotas influenciam diretamente nos parâmetros avaliados. Conclui-se, portanto, o número mínimo de cartões de papel hidrossensível por repetição $\left(1 \mathrm{~m}^{2}\right.$ no caso deste experimento) em experimentações é de 7,5 e 1 para as variáveis percentual de cobertura, número de gotas por $\mathrm{cm}^{2}$ e diâmetro mediano volumétrico, respectivamente.

Palavras-chave: Número de amostras, cobertura, ponta de pulverização, taxa de aplicação

\begin{abstract}
The raise in the numbers of samples increase the confidence level of the results. However little is know about the minimum number of water-sensitive papers cards that must be used in studies of spray technology. Thus, the aim of this paper was to determine the minimum number of water-sensitive papers cards used in spray experiments, based on the variables: percentage of coverage, number of drops per $\mathrm{cm}^{2}$ and volume median diameter (VMD). It were realized an experiment in factorial design $2 \times 2$ with five replicates, each containing 36 cards. With the objective to have variability among the treatments,

${ }^{1}$ Eng $^{\circ}$ Agr $^{\circ}$, M.e em Agronomia, Universidade Estadual de Londrina, UEL, Londrina, PR. E-mail: ballarotti@hotmail.com

${ }^{2}$ Eng $^{\circ}$ Agr $^{\circ}$, Discente de Doutorando em Agronomia, UEL, Londrina, PR. E-mail: gmigliorini@hotmail.com

${ }^{3}$ Eng $^{{ }^{\mathrm{s}}}$ Agr $^{\text {ss }}$, Profs. Drs. Adjunto, Dept ${ }^{\circ}$ de Agronomia, UEL, Londrina, PR. E-mail: inescbf@uel.br; abisaab@uel.br; canteri@uel.br
\end{abstract}

* Autor para correspondência 
the factors involved were nozzles (TR110-01 and ULD120-015) and operational pressures (138 and $276 \mathrm{kPa}$ ), because the size of droplets is modified even by the nozzle as by operational pressures. As estimative precision, the mean errors assumed was $20 \%, 15 \%$ and $10 \%$, that generated numbers of samples as: 7, 12 and 26, respectively, for percentage of coverage; 5, 8 and 16, respectively, for number of drops per $\mathrm{cm}^{2}$; and 1.2 and 4 respectively, for volume median diameter (VMD). Analysis of variance complemented by Tukey test at $5 \%$ probability were realized for the average of 36 cards and the average of the minimum number of papers (samples) determined. Comparing statically the averages obtained in the experiment for each variable for $\mathrm{n}=36$ samples and $\mathrm{n}=$ samples determined at $20 \%$ error, noted that the results are identical in both situations, demonstrating that spray rates and different droplet sizes influenced directly in the parameters evaluated. It is possible to conclude that the minimum number of water-sensitive papers cards per repetition ( $1 \mathrm{~m}^{2}$ for this experiment) is 7,5, and 1 for percentage of coverage, number of droplet per $\mathrm{cm}^{2}$ and volume median diameter (VMD), respectively.

Key words: Number of samples, percentage of coverage, spray nozzles, application rates

\section{Introdução}

De todas as formas de controle dos agentes nocivos às culturas, o uso de produtos fitossanitários tem sido o principal método de controle, sendo a maior parte destes produtos aplicados por via líquida. A aplicação fitossanitária via líquida consiste na colocação das gotas pulverizadas sobre o alvo. Porém, tal processo é conhecido pela sua baixa eficiência, por não se conseguir atingir o alvo de forma uniforme, podendo resultar, muitas vezes, em um controle inadequado, com desperdício de produtos fitossanitários e contaminação ambiental.

A pulverização pode ocorrer pelo uso de bicos hidráulicos, bicos pneumáticos, termonebulizadores e por discos centrífugos (VASQUEZ, 2004). Com bicos hidráulicos é a forma mais comum de pulverizar. O processo de formação de gotas se inicia na saída do líquido sob pressão pela ponta de pulverização. A energia potencial do líquido, associada com o desenho da ponta e geometria do orifício faz o líquido emergir em pequenos filetes. Esses filetes posteriormente quebram-se em pequenas peças, que são chamadas de gotas, gotículas ou partículas líquidas (SCHICK, 1997).

Atualmente existem inúmeras pontos que podem ser manejados quando se pensa na realização de uma aplicação via líquida, como por exemplo, a taxa de aplicação $\left(\mathrm{L} . h a^{-1}\right)$, tamanho de gotas pulverizadas, assistência de ar, eletrificação das gotas, uso de adjuvantes na calda de aplicação, entre outros.
Em tais aplicações, o padrão de distribuição e a uniformidade de cobertura das gotas sobre o alvo são diretamente relacionados com a eficiência da pulverização e eficácia no controle.

O percentual de cobertura proporcionado por estas gotas sobre o alvo pode ser influenciado por diversos fatores, tais como condições climáticas, velocidade de aplicação, tamanho de gotas, arquitetura da planta alvo e de outras características do próprio pulverizador.

Uma das formas de avaliar a cobertura proporcionada por uma aplicação é pelo uso do cartão de papel hidrossensível, que mostra a quantidade e a característica das gotas depositadas sobre o mesmo, podendo ser uma indicação relativamente precisa e barata da qualidade de aplicação, sobretudo de fácil utilização.

A principal característica dos cartões de papel hidrossensível são papéis semi-rígidos, com uma camada composta de azul de bromofenol, refletindo a cor amarela, que se torna azul quando em contato com a água, devido a mudança de $\mathrm{pH}$ de 2,8 para 4,6 (TURNER; HUNTINGTON, 1970). Esses cartões são bastante populares em todo o mundo, sendo distribuídos pelos principais fabricantes de pontas de pulverização e produzidos em larga escala desde 1985 (PARKIN, 1993).

Uma característica dos cartões de papel hidrossensível é que as gotas pulverizadas que o atingem causam manchas no mesmo. A determinação 
da porcentagem de cobertura e/ou número de gotas que atingem os papéis por área pode ser feita manualmente, contando-se visualmente as gotas contidas em espaço determinado, por exemplo, $1 \mathrm{~cm}^{2}$ ou através de softwares específicos para análise das imagem dos impactos ou manchas no papel, podendose calcular deste modo também o tamanho das gotas, ou seja, o diâmetro mediano volumétrico (DMV).

A alta umidade relativa do ar, acima de $85 \%$, faz com que toda superfície dos cartões de papel hidrossensível se torne azul, impossibilitando seu uso. Outro fator limitante é a interpolação das gotas sobre o papel que podem causar manchas contínuas que distorcem a interpretação do tamanho das gotas, ao ponto de impossibilitar o cálculo do número de impactos por área e seu tamanho, principalmente em aplicações com elevadas taxas de aplicação (FOX et al., 2001). Por esta razão Degré et al. (2001) consideraram que os cartões de papel hidrossensível tiveram boa indicação qualitativa da aplicação em volumes não maiores que 100 L.ha-1 . Outra limitação técnica ao uso de softwares de análise de gotas em papéis sensíveis se deve a dificuldade de leitura e análise de gotas muito pequenas, abaixo de 50 micra (HOFFMAN; HEWITT, 2005).

Salyani e Fox (1994) foram pioneiros na utilização de computadores na análise de manchas simuladas por impressora a jato de tinta. O trabalho, entretanto, teve sucesso restrito devido a limitações de desempenho da impressora e do próprio software, visto que os equipamentos disponíveis na época eram bastante rudimentares, comparados aos atuais.

Diversos outros trabalhos abordaram a leitura e interpretação de cartões de papel hidrossensível (PANNETON, 2002; PESSOA; CHAIM, 1999). Entretanto, pouco se conhece sobre a variabilidade da deposição de tais gotas em experimentos ligados à tecnologia de aplicação, bem como qual seria a quantidade ideal ou mínima de cartões de papel hidrossensível a serem utilizados em tais experimentações.

A determinação do número mínimo de cartões de papel hidrossensível, ou seja, do tamanho da amostra, depende basicamente da variação dos dados de uma determinada variável quantitativa, bem como a margem de erro assumida para sua base de cálculo, em que quanto maior a variação dos dados e menor a margem de erro assumida, maior é o número de amostras requeridas (ZAR, 1999). Assim a condição em que é feito o estudo aliado ainda à segurança e/ou a aplicação prática do resultado é de suma importância para o estabelecimento da margem de erro estudada.

O objetivo deste trabalho foi determinar o número mínimo de cartões de papel hidrossensível por área $\left(\mathrm{m}^{2}\right)$ a serem amostrados em um experimento, envolvendo pontas de pulverização e pressão de trabalho, tendo como base as variáveis: cobertura porcentual, cobertura numérica e diâmetro mediano volumétrico (DMV).

\section{Material e Métodos}

$\mathrm{O}$ experimento foi realizado nas dependências do Centro de Ciências Agrárias da Universidade Estadual de Londrina. Foram realizadas aplicações em pulverização com água sobre cartões de papel hidrossensível, dispostos sobre superfície plana, por meio do uso de um equipamento costal, pressurizado por $\mathrm{CO}_{2}$, com barra de pulverização contendo quatro bicos espaçados de $0,5 \mathrm{~m}$.

Nas aplicações, feitas sempre pelo mesmo operador do equipamento, a barra de pulverização foi disposta centralizada em relação à parcela, mantida a 0,5 $\mathrm{m}$ de altura desta, e em ambiente fechado para isolar o efeito do vento. A umidade relativa e a temperatura do ar foram monitoradas durante as aplicações, através de termohigrômetro, variando de 71 a $75 \%$ e 26,0 a $26,7^{\circ} \mathrm{C}$, respectivamente.

$\mathrm{O}$ delineamento experimental foi inteiramente casualizado, em esquema fatorial 2 × 2 ( 2 pontas de pulverização TK 11001 e ULD 120015 e 2 pressões de trabalho 138 e $276 \mathrm{kPa}$ ), com 5 repetições. Cada uma das 20 parcelas constitui-se 
de 36 cartões de papel hidrossensível, dispostos de forma padronizada em $1 \mathrm{~m}^{2}$ de superfície plana sobre o solo, sendo 18 em posição longitudinal e 18 em posição transversal ao sentido de deslocamento da barra de pulverização, totalizando 720 unidades.
A velocidade aproximada de trabalho foi de $6 \mathrm{~km} \cdot \mathrm{h}^{-1}$. As características das pontas utilizadas, assim como as respectivas vazões e tamanho de gotas geradas nas diferentes pressões de trabalho utilizadas, estão apresentadas na Tabela 1.

Tabela. 1. Características das pontas de pulverização utilizadas nas respectivas pressões de trabalho estudadas.

\begin{tabular}{|c|c|c|c|c|c|c|}
\hline \multirow{3}{*}{ Modelo da Ponta } & \multicolumn{6}{|c|}{ Pressão de trabalho $(\mathrm{kPa})$} \\
\hline & \multicolumn{3}{|c|}{138} & \multicolumn{3}{|c|}{276} \\
\hline & $\begin{array}{c}\text { Tamanho } \\
\text { de gota }\end{array}$ & $\begin{array}{c}\text { Vazão } \\
\left(\text { L.min }{ }^{-1}\right)\end{array}$ & $\begin{array}{l}\text { Taxa de aplicação } \\
\left(\text { L.ha }^{-1}\right)\end{array}$ & $\begin{array}{l}\text { Tamanho } \\
\text { de gota }\end{array}$ & $\begin{array}{c}\text { Vazão } \\
\left(\text { L.min }{ }^{-1}\right)\end{array}$ & $\begin{array}{l}\text { Taxa de aplicação } \\
\left(\text { L.ha }^{-1}\right)\end{array}$ \\
\hline $\begin{array}{l}\text { TR110-01 (Jato plano } \\
\text { padrão) }\end{array}$ & Fina & 0,25 & 50 & Muito fina & 0,35 & 70 \\
\hline $\begin{array}{l}\text { ULD120-015 (Jato plano } \\
\text { com indução de ar) }\end{array}$ & Grossa & 0,39 & 78 & Média & 0,525 & 105 \\
\hline
\end{tabular}

Fonte: Elaboração dos autores.

Após as aplicações os cartões de papel hidrossensível foram embalados em filme plástico de PVC transparente, a fim de preservá-las da contaminação pela umidade do ar, sendo cada um digitalizado em um aparelho scanner de mesa, com resolução de 300 dpi. As imagens foram processadas, através do uso do software ContaGotas (CANTERI et al., 2001), para a estimativa da porcentagem de cobertura, número de gotas por área e (DMV).

A determinação da quantidade mínima de papéis hidrossensíveis (amostras), para cada variável (cobertura, gotas por $\mathrm{cm}^{2}$ e DMV) foi calculado, para cada tratamento em específico pontas e pressões, com base na equação descrita (ZAR, 1999) abaixo:

$$
\mathrm{n}=\frac{\mathrm{t}^{2} \times \mathrm{S}^{2}}{\mathrm{~d}^{2}}
$$

em que:

$\mathrm{n}=$ tamanho da amostras;

$\mathrm{t}=$ valor amostral tabelado ( $\mathrm{t}$ de Student $)(\alpha=$ 0,05 e graus de liberdade $=35$ );
$\mathrm{S}=$ desvio padrão;

$\mathrm{d}=$ erro porcentual em relação à média.

Para a comparação e validação dos resultados obtidos foram realizadas as análises de variância, seguidas do teste Tukey em nível de 5\% de probabilidade, com os valores das médias dos 36 cartões de papel hidrossensível por repetição, bem como para a média utilizando o número mínimo de cartões por amostras determinado pela equação anterior. Nas analises assumiu-se o erro de $20 \%$, $15 \%$ e $10 \%$, para a determinação do número mínimo de amostras de cartões de papel hidrossensível nas três variáveis estudadas (porcentual de cobertura, número de gotas por $\mathrm{cm}^{2} \mathrm{e} \mathrm{DMV}$ ), calculados para cada repetição de cada tratamento.

\section{Resultados e Discussão}

Para o percentual de cobertura verificouse que o número mínimo de cartões de papel hidrossensível para os erros de $20 \%, 15 \%$ e 10\% foram: $7(6,44) ; 12(11,45)$ e $26(25,76)$, respectivamente (Tabela 2$)$. 
Tabela 2. Média, coeficiente de variação (CV\%) e números de amostras requeridas (n) para $20 \%, 15 \%$, e $10 \%$ de margem de erro quanto à cobertura porcentual.

\begin{tabular}{|c|c|c|c|c|c|c|c|}
\hline Ponta & Pressão & Repetição & Média * & $\mathrm{CV}(\%)$ & $\mathbf{n}_{20 \%}$ & $\mathbf{n}_{15 \%}$ & $\mathbf{n}_{10 \%}$ \\
\hline \multirow{10}{*}{ TR 110-01 } & \multirow{5}{*}{$138 \mathrm{kPa}$} & 1 & 16,83 & 19,6 & 3,96 & 7,04 & 15,83 \\
\hline & & 2 & 18,33 & 20,33 & 4,26 & 7,57 & 17,02 \\
\hline & & 3 & 19 & 15,71 & 2,54 & 4,52 & 10,18 \\
\hline & & 4 & 18,75 & 12,32 & 1,56 & 2,78 & 6,25 \\
\hline & & 5 & 19,31 & 14,64 & 2,21 & 3,93 & 8,83 \\
\hline & \multirow{5}{*}{$276 \mathrm{kPa}$} & 1 & 16,17 & 19,31 & 3,84 & 6,83 & 15,36 \\
\hline & & 2 & 16,47 & 17,26 & 3,07 & 5,46 & 12,28 \\
\hline & & 3 & 16,58 & 16,71 & 2,88 & 5,11 & 11,51 \\
\hline & & 4 & 15,92 & 14,04 & 2,03 & 3,61 & 8,12 \\
\hline & & 5 & 16,44 & 18,41 & 3,49 & 6,21 & 13,97 \\
\hline \multirow{10}{*}{ ULD 120-015 } & \multirow{5}{*}{$138 \mathrm{kPa}$} & 1 & 22,06 & 18,04 & 3,35 & 5,96 & 13,41 \\
\hline & & 2 & 18,42 & 21,62 & 4,81 & 8,56 & 19,26 \\
\hline & & 3 & 19,28 & 25 & $6,44 * *$ & $11,45^{* *}$ & $25,76 * *$ \\
\hline & & 4 & 19,75 & 23,61 & 5,74 & 10,21 & 22,96 \\
\hline & & 5 & 15,72 & 21,96 & 4,97 & 8,83 & 19,87 \\
\hline & \multirow{5}{*}{$276 \mathrm{kPa}$} & 1 & 34,06 & 9,28 & 0,89 & 1,58 & 3,55 \\
\hline & & 2 & 33,14 & 6,62 & 0,45 & 0,8 & 1,8 \\
\hline & & 3 & 33,28 & 6,91 & 0,49 & 0,88 & 1,97 \\
\hline & & 4 & 32,94 & 4,86 & 0,24 & 0,43 & 0,98 \\
\hline & & 5 & 33,03 & 4,88 & 0,25 & 0,44 & 0,98 \\
\hline
\end{tabular}

*Médias de 36 papéis hidrossensíveis (amostras). **Destaques (em negrito) referem-se aos maiores valores de $\mathrm{n}$ para cada margem de erro.

Fonte: Elaboração dos autores.

$\mathrm{Na}$ comparação das análises de variância do encontrados foram idênticos, ocorrendo interação fatorial ponta $\mathrm{x}$ pressão, tanto para médias de $\mathrm{n}=$ significativa entre os fatores estudados nas duas 36 e n $=7$ (número amostral mínimo), os resultados situações de análise (Tabela 3).

Tabela 3. Cobertura porcentual das pontas de pulverização TR110-01 e ULD-120-015, sob diferentes pressões de trabalho, para médias de $n=36$ e $n=7$ papéis hidrossensíveis.

\begin{tabular}{ccc}
\hline \multicolumn{3}{c}{ Porcentagem de cobertura (\%) - n=36 papéis hidrossensíveis } \\
\hline \multicolumn{3}{c}{ Ponta de Pulverização } \\
Pressão de trabalho & TR 110-01 & ULD 120-015 \\
$138 \mathrm{kPa}$ & $18,44 \mathrm{Aa}$ & $19,04 \mathrm{Ba}$ \\
$276 \mathrm{kPa}$ & $16,31 \mathrm{Bb}$ & $33,29 \mathrm{Aa}$ \\
\hline $\mathrm{CV}(\%)$ & \multicolumn{3}{c}{5,85} \\
\hline \multicolumn{3}{c}{ Porcentagem de cobertura (\%) - n=7 papéis hidrossensíveis } \\
\hline \multicolumn{3}{c}{ Ponta de pulverização } \\
Pressão de trabalho & ULD 120-015 \\
$138 \mathrm{kPa}$ & $18,60 \mathrm{Aa}$ & $19,45 \mathrm{Ba}$ \\
$276 \mathrm{kPa}$ & $15,57 \mathrm{Bb}$ & $33,28 \mathrm{Aa}$ \\
\hline $\mathrm{CV}(\%)$ & \multicolumn{3}{c}{5,63} \\
\hline
\end{tabular}

Médias seguidas de mesma letra, maiúsculas nas colunas e minúsculas nas linhas, não diferem entre si pelo teste Tukey à 5\% de probabilidade.

Fonte: Elaboração dos autores. 
O resultado do desdobramento das interações em ambas as analises $(n=36$ e $n=7)$ evidenciaram que a vazão $\left(\right.$ L. min $^{-1}$ ) e o tamanho de gotas proporcionadas pelas diferentes pontas durante a pulverização foram determinantes para o percentual de cobertura encontrada. A ponta ULD120-015, que promoveu as maiores taxas de aplicação (78 e 105 L.ha $^{-1}$ ) e gotas maiores (grossas e médias, respectivamente) nas duas pressões de trabalho adotadas, apresentou a mesma cobertura porcentual do que a ponta TR110-01, quando submetida a pressão de trabalho de $138 \mathrm{kPa}$ (taxa de aplicação de 50 L.ha-1 e gotas finas). O aumento na pressão de trabalho para 276 $\mathrm{kPa}$ fez com que a ponta TR110-01 aumentasse a taxa de aplicação de 50 para 70 L.ha-1 ${ }^{-1}$ reduzindo, porém, o tamanho de gota de fina para muito fina. No entanto, apesar da taxa ser maior, a cobertura foi menor. Isso pode ter ocorrido em função de perdas por evaporação e deriva, ou pelo fato de gotas menores que 100 micra não serem detectadas satisfatoriamente pelo software de leitura da imagem no papel sensível, conforme Degré et al. (2001).

O aumento na taxa de aplicação, assim como a redução do tamanho de gotas, teoricamente promove melhoria no nível de cobertura em função do maior número de gotas pulverizadas até o alvo. Porém, gotas muito pequenas estão mais sujeitas aos fatores climáticos, podendo ser desviadas e/ ou seu volume ser evaporado ao longo de seu deslocamento até o alvo, não atingindo muito vezes o objetivo (CUNHA; TEIXEIRA, 2001; VIANA et al., 2008).

Quanto ao número de gotas por área, o número mínimo de cartões de papel hidrossensível encontrados, para os erros de 20\%, 15\% e 10\% foram: $5(4,15) ; 8(7,38)$ e $17(16,60)$, respectivamente (Tabela 4 ).

Tabela 4. Média, coeficiente de variação (CV\%) e números de amostras requeridas (n) para 20\%, 15\%, e $10 \%$ de margem de erro quanto ao número de gotas por área.

\begin{tabular}{|c|c|c|c|c|c|c|c|}
\hline Ponta & Pressão & Repetição & Média * & CV(\%) & $\mathbf{n}_{20 \%}$ & $\mathbf{n}_{15 \%}$ & $n_{10 \%}$ \\
\hline \multirow{10}{*}{ TR 110-01 } & \multirow{5}{*}{$138 \mathrm{kPa}$} & 1 & 105,33 & 6,49 & 0,43 & 0,77 & 1,74 \\
\hline & & 2 & 105,89 & 7,79 & 0,62 & 1,11 & 2,5 \\
\hline & & 3 & 107,61 & 5,98 & 0,37 & 0,65 & 1,47 \\
\hline & & 4 & 108,5 & 4,16 & 0,18 & 0,32 & 0,71 \\
\hline & & 5 & 108,64 & 5,2 & 0,28 & 0,49 & 1,11 \\
\hline & \multirow{5}{*}{$276 \mathrm{kPa}$} & 1 & 117,39 & 4,94 & 0,25 & 0,45 & 1,01 \\
\hline & & 2 & 117,72 & 5,51 & 0,31 & 0,56 & 1,25 \\
\hline & & 3 & 116,03 & 3,32 & 0,11 & 0,2 & 0,45 \\
\hline & & 4 & 116,25 & 2,78 & 0,08 & 0,14 & 0,32 \\
\hline & & 5 & 116,72 & 3,77 & 0,15 & 0,26 & 0,59 \\
\hline \multirow{10}{*}{ ULD 120-015 } & \multirow{5}{*}{$138 \mathrm{kPa}$} & 1 & 49,64 & 15,49 & 2,47 & 4,4 & 9,89 \\
\hline & & 2 & 45,22 & 14,74 & 2,24 & 3,98 & 8,95 \\
\hline & & 3 & 46,61 & 18,65 & 3,58 & 6,37 & 14,33 \\
\hline & & 4 & 46,81 & 20,07 & $4,15^{* *}$ & $7,38 * *$ & $16,60 * *$ \\
\hline & & 5 & 40,28 & 18,75 & 3,62 & 6,44 & 14,5 \\
\hline & \multirow{5}{*}{$276 \mathrm{kPa}$} & 1 & 86,36 & 2,2 & 0,05 & 0,09 & 0,2 \\
\hline & & 2 & 85,92 & 2,16 & 0,05 & 0,09 & 0,19 \\
\hline & & 3 & 85,31 & 2,44 & 0,06 & 0,11 & 0,25 \\
\hline & & 4 & 84,64 & 2,78 & 0,08 & 0,14 & 0,32 \\
\hline & & 5 & 84,83 & 3,33 & 0,11 & 0,2 & 0,46 \\
\hline
\end{tabular}

*Médias de 36 papéis hidrossensíveis (amostras). **Destaques (em negrito) referem-se aos maiores valores de $\mathrm{n}$ para cada margem de erro.

Fonte: Elaboração dos autores. 
Novamente houve interação significativa entre os fatores ponta x pressão, para o resultado da análise de variância das médias de ambos os números de cartões de papel hidrossensível $(\mathrm{n}=36$ e $\mathrm{n}=5$, número amostral mínimo). Os resultados encontrados foram semelhantes em ambas as análises (Tabela 5).

Tabela 5. Número de gotas por área entre as pontas de pulverização TR110-01 e ULD-120-015, sob diferentes pressões de trabalho, para médias de $n=36$ e $n=5$ papéis hidrossensíveis.

\begin{tabular}{|c|c|c|}
\hline \multicolumn{3}{|c|}{ Número de gotas por $\mathrm{cm}^{2}-\mathrm{n}=36$ papéis hidrossensíveis } \\
\hline \multirow{2}{*}{ Pressão de trabalho } & \multicolumn{2}{|c|}{ Ponta de pulverização } \\
\hline & TR 110-01 & ULD 120-015 \\
\hline $138 \mathrm{kPa}$ & $107,19 \mathrm{Ba}$ & $45,71 \mathrm{Bb}$ \\
\hline $276 \mathrm{kPa}$ & $116,82 \mathrm{Aa}$ & $85,41 \mathrm{Ab}$ \\
\hline $\mathrm{CV}(\%)$ & & \\
\hline \multicolumn{3}{|c|}{ Número de gotas por $\mathrm{cm}^{2}-\mathrm{n}=5$ papéis hidrossensíveis } \\
\hline \multirow{2}{*}{ Pressão de trabalho } & \multicolumn{2}{|c|}{ Ponta de pulverização } \\
\hline & TR $110-01$ & ULD 120-015 \\
\hline $138 \mathrm{kPa}$ & $108,52 \mathrm{Ba}$ & $46,76 \mathrm{Bb}$ \\
\hline $276 \mathrm{kPa}$ & $116,96 \mathrm{Aa}$ & $85,36 \mathrm{Ab}$ \\
\hline $\mathrm{CV}(\%)$ & & \\
\hline
\end{tabular}

Médias seguidas de mesma letra, maiúsculas nas colunas e minúsculas nas linhas, não diferem entre si pelo teste Tukey à 5\% de probabilidade.

Fonte: Elaboração dos autores.

Os resultados referentes aos fatores isolados (ponta e pressão) para ambas as analise $(\mathrm{n}=36$ e $\mathrm{n}=5$ ) mostram que o aumento na pressão de trabalho, de $138 \mathrm{kPa}$ para $276 \mathrm{kPa}$, assim como o uso da ponta TR 110-01 em relação à ULD-120015, proporcionaram maior número de gotas por área, independentemente da interação entre os dois fatores. Esses resultados já eram esperados, uma vez que o aumento da pressão de trabalho promove, além do aumento da vazão, a diminuição do tamanho (volume) das gotas, ou seja, o aumento do número total de gotas produzidas. Assim como, era esperado que a ponta que produz gotas de tamanho menor (TR 110-01) também produzisse um número maior dessas, em comparação à ponta ULD-120-015

O DMV, por sua vez, foi a variável que apresentou os menores números de cartões de papel hidrossensível para os erros de $20 \% 15 \%$ e $10 \%$ - $1(0,96), 2(1,72)$ e $4(3,86)$, respectivamente (Tabela 6). 
Tabela 6. Média, coeficiente de variação (CV\%) e números de amostras requeridas (n) para $20 \%, 15 \%$, e $10 \%$ de margem de erro quanto ao diâmetro mediano volumétrico (DMV).

\begin{tabular}{|c|c|c|c|c|c|c|c|}
\hline Ponta & Pressão & Repetição & Média* & CV(\%) & $\mathrm{n}_{20 \%}$ & $\mathbf{n}_{15 \%}$ & $\mathbf{n}_{10 \%}$ \\
\hline \multirow{10}{*}{ TR 110-01 } & \multirow{5}{*}{$138 \mathrm{kPa}$} & 1 & 295,39 & 7,14 & 0,53 & 0,93 & 2,1 \\
\hline & & 2 & 301,61 & 6,55 & 0,44 & 0,79 & 1,77 \\
\hline & & 3 & 305,58 & 4,71 & 0,23 & 0,41 & 0,92 \\
\hline & & 4 & 307,08 & 3,54 & 0,13 & 0,23 & 0,52 \\
\hline & & 5 & 305,19 & 5,3 & 0,29 & 0,52 & 1,16 \\
\hline & \multirow{5}{*}{$276 \mathrm{kPa}$} & 1 & 217,69 & 2,72 & 0,08 & 0,14 & 0,31 \\
\hline & & 2 & 216,78 & 1,98 & 0,04 & 0,07 & 0,16 \\
\hline & & 3 & 216,17 & 2,49 & 0,06 & 0,11 & 0,26 \\
\hline & & 4 & 215,22 & 2,28 & 0,05 & 0,1 & 0,21 \\
\hline & & 5 & 217,72 & 2,18 & 0,05 & 0,09 & 0,2 \\
\hline \multirow{10}{*}{ ULD 120-015 } & \multirow{5}{*}{$138 \mathrm{kPa}$} & 1 & 611,39 & 8,36 & 0,72 & 1,28 & 2,88 \\
\hline & & 2 & 586,06 & 6,97 & 0,5 & 0,89 & 2 \\
\hline & & 3 & 601,08 & 8,19 & 0,69 & 1,23 & 2,76 \\
\hline & & 4 & 606,08 & 9,68 & $0,96 * *$ & $1,72 * *$ & $3,86 * *$ \\
\hline & & 5 & 563,39 & 7,17 & 0,53 & 0,94 & 2,12 \\
\hline & \multirow{5}{*}{$276 \mathrm{kPa}$} & 1 & 474,39 & 1,8 & 0,03 & 0,06 & 0,13 \\
\hline & & 2 & 472,11 & 1,81 & 0,03 & 0,06 & 0,13 \\
\hline & & 3 & 475,03 & 1,99 & 0,04 & 0,07 & 0,16 \\
\hline & & 4 & 473,94 & 1,79 & 0,03 & 0,06 & 0,13 \\
\hline & & 5 & 477,47 & 1,9 & 0,04 & 0,07 & 0,15 \\
\hline
\end{tabular}

*Médias de 36 papéis hidrossensíveis (amostras). **Destaques (em negrito) referem-se aos maiores valores de $\mathrm{n}$ para cada margem de erro.

Fonte: Elaboração dos autores.

Houve novamente interação significativa entre variâncias relativas aos números de cartões de papel os fatores ponta x pressão em ambas as análises de hidrossensível amostrados, $n=36$ e $n=1$ (Tabela 7).

Tabela 7. Diâmetro mediano volumétrico (DMV) entre as pontas de pulverização (TR110-01 e ULD-120-015) sob diferentes pressões de trabalho ( 138 e $276 \mathrm{kPa}$ ), para médias de $n=36$ e $\mathrm{n}=5$ papéis hidrossensíveis.

\begin{tabular}{|c|c|c|}
\hline \multicolumn{3}{|c|}{ Diâmetro mediano volumétrico $(\mathrm{DMV})-\mathrm{n}=36$ papéis hidrossensíveis } \\
\hline \multirow{2}{*}{ Pressão de trabalho } & \multicolumn{2}{|c|}{ Ponta de pulverização } \\
\hline & TR $110-01$ & ULD $120-015$ \\
\hline $138 \mathrm{kPa}$ & $302,97 \mathrm{Ab}$ & $593,60 \mathrm{Aa}$ \\
\hline $276 \mathrm{kPa}$ & $216,71 \mathrm{Bb}$ & $474,58 \mathrm{Ba}$ \\
\hline $\mathrm{CV}(\%)$ & & \\
\hline \multicolumn{3}{|c|}{ Diâmetro mediano volumétrico (DMV) - n = 1 papel hidrossensível } \\
\hline \multirow{2}{*}{ Pressão de trabalho } & \multicolumn{2}{|c|}{ Ponta de pulverização } \\
\hline & TR $110-01$ & ULD $120-015$ \\
\hline $138 \mathrm{kPa}$ & $299,00 \mathrm{Ab}$ & $614,80 \mathrm{Aa}$ \\
\hline $276 \mathrm{kPa}$ & $218,60 \mathrm{Bb}$ & $473,40 \mathrm{Ba}$ \\
\hline $\mathrm{CV}(\%)$ & & \\
\hline
\end{tabular}

Médias seguidas de mesma letra, maiúsculas nas colunas e minúsculas nas linhas, não diferem entre si pelo teste Tukey à 5\% de probabilidade.

Fonte: Elaboração dos autores. 
O resultado do desdobramento das analises $(\mathrm{n}=$ 36 e $n=1$ ) confirmam a informação do fabricante de que, nas pressões de trabalho utilizadas, a ponta TR110-01 produz gotas menores (finas e muito finas) do que a ponta ULD120-015 (grossas e médias). Assim como, confirmam que com o aumento da pressão diminui o tamanho da gota.

Considerando a baixa variabilidade dos dados dentro de cada tratamento, o que possibilitou conseqüentemente menor número de amostras necessárias para resultados confiáveis, o cartão de papel hidrossensível pode ser uma ferramenta bastante importante na comparação das variáveis (percentual de cobertura, número de gotas por $\mathrm{cm}^{2}$ e diâmetro mediano volumétrico), geradas pelas gotas oriundas de pontas de pulverização.

Mantendo-se as mesmas condições do estudo, os resultados mostraram que na amostra, o número de cartões de papel hidrossensível por parcela poderia ser reduzida de 36 para 7, 5 e 1 para as variáveis cobertura porcentual, número de gotas por área e DMV, respectivamente. Contudo, cabe salientar que para a correta interpretação dos resultados obtidos em pulverizações agrícolas a partir de cartões de papel hidrossensível, inúmeros fatores devem ser considerados, pois estes sofrem influência da:

- Umidade relativa do ar: o seu uso em condições de alta umidade relativa do ar pode ser comprometido por contaminação das amostras, que mudam a sua cor de amarela para azul em função do contato com a água da atmosfera (TURNER; HUNTINGTON, 1970).

- Características da calda: a adição de adjuvantes, ou mesmo de produtos fitossanitários, modificam a tensão superficial da água e podem alterar o fator de espalhamento das gotas, fazendo com que valores específicos sejam necessários para utilizar os programas de leitura (FRITZ et al., 2009).

- Softwares: diferentes programas de leitura podem gerar diferentes estimativas (LEIVA; ARAUJO, 2007);

- Taxas de aplicação elevadas: devido à coalescência de gotas, causando manchas contínuas, distorcendo a estimativa do tamanho de gotas, como também do número de impactos por área (FOX et al., 2001; FRITZ et al., 2009). Por essa razão Degré et al. (2001) sugerem que o uso de cartões de papel hidrossensível não deva ser utilizado em taxas de aplicações superiores a 100 L.ha $^{-1}$;

- Gotas muito pequenas: dificuldade de leitura pelos softwares de gotas muito pequenas, abaixo de 50 micra (HOFFMAN; HEWITT, 2005) ou 80 micra (SALYANI; FOX, 1999);

- Alvo artificial: por se tratar de um alvo artificial, que não tem as mesmas propriedades morfo-anatômicas dos alvos naturais que são em sua grande maioria as folhas das culturas, os resultados encontrados geram apenas inferências acerca dos fatos ocorridos durante uma aplicação.

Embora alguns fatores sejam limitantes para determinados tipos de estudos, a utilização de cartões de papel hidrossensível em experimentos ligados a tecnologia de aplicação vem sendo cada vez mais difundida entre os pesquisadores e extensionistas de diferentes áreas atreladas ao controle químico como um todo na agricultura, trazendo consigo importantes informações a respeito dos fatores interligados ao controle de plantas daninhas, pragas e doenças.

Um exemplo de grande e atual importância do uso destes cartões de papel hidrossensível é o estudo relacionado à tecnologia de aplicação e/ou controle químico da ferrugem asiática da soja (Pkakopsora pachyrhizi) na última década em função da ocorrência epidêmica em determinadas regiões (OZKHAN; ZHU; DERKSEN, 2006; CUNHA et al., 2008; ROMAN, et al., 2009). Tais trabalhos efetuados com papéis hidrossensíveis têm gerado inúmeras informações relevantes quanto aos fatores inerentes à penetração das gotas no dossel inferior da cultura, local em que se inicia o desenvolvimento da doença e que deve, por conseguinte, ter máxima ação dos fungicidas, promovendo assim um aumento substancial no entendimento dos fatores relativos a tecnologia de aplicação e a eficácia de controle. 


\section{Conclusão}

Considerando os resultados nas condições em que foi desenvolvido o estudo pode-se concluir que o número mínimo de cartões de papel hidrossensível por repetição $\left(1 \mathrm{~m}^{2}\right.$ no caso deste experimento) em experimentações é de 7,5 e 1 para as variáveis percentual de cobertura, número de gotas por $\mathrm{cm}^{2}$ e DMV, respectivamente. Para a validação em diferentes condições das realizadas neste estudo, este trabalho deve ser repetido com outros equipamentos, pontas de pulverização, pressões de trabalho, etc.

\section{Referências}

CANTERI, M. G.; FÜRSTENBERGER, A. L. F.; GARCIA, L. C.; JUSTINO, A. Conta-gotas: sistema para análise de eficiência de pulverização. In: CONGRESSO PAULISTA DE FITOPATOLOGIA, 27., 2001. Piracicaba, SP. Anais... Jaboticabal: Grupo Paulista de Fitopatologia, 2001, p. 136.

CUNHA, J. P. A. R.; MOURA, E. A. C.; SILVA JUNIOR, J. L. da; ZAGO, F. A.; JULIATTI, F. C. Efeito de pontas de pulverização no controle químico da ferrugem da soja. Engenharia Agrícola, Jaboticabal, v. 28, n. 2, p. 283-291, 2008.

CUNHA, J. P. A. R.; TEIXEIRA, M. M. Características técnicas de bicos de pulverização hidráulicos de jato plano. Revista Brasileira de Engenharia Agrícola e Ambiental, Campina Grande, v. 5, n. 2, p. 344-348, 2001.

DEGRÉ, A.; MOSTADE, B.; HUYGHEBAERT, S.; TISSOT, S.; DEBOUCHE, C. Comparison by image processing of target supports of spray droplets. ASABE - Transaction of ASAE, St. Joseph, MI, v. 44, n. 2, p. 217 222, 2001.

FOX, R. D.; SALYANI, M.; COOPER, J. A.; BRAZEE, R. D. Spot size comparisons on oil- and water-sensitive paper. ASABE - Applied Engineering in Agriculture, St. Joseph, MI, v. 17, n. 2, p. 131-136, 2001.

FRITZ, B. K.; PARKER, J. D.; LÓPEZ JUNIOR, J. D.; HOFFMAN, W. C.; SCHLEIDER, P. Deposition and droplet sizing characterization of a laboratory spray table. ASABE - Applied Engineering in Agriculture, St. Joseph, MI, v. 25, n. 2, p. 175-180, 2009.

HOFFMAN, W. C.; HEWITT, A. J. Comparison of three imaging systems for water sensitive papers. ASABE, Applied Engineering in Agriculture, St. Joseph, MI, v.
21, n. 6, p. 961-964, 2005.

LEIVA, P. D.; ARAÚJO, E. C. Comparación de programas de computación para recuento y tipificación de impactos de aspersión sobre tarjetas sensibles. 2007. Disponível em: <http://www.agrotec.etc.br/downloads/ Comparacion_de_programas_de_computacion_para recuento.pdf >. Acesso em: 05 dez. 2010.

OZKHAN, H. E.; ZHU, H.; DERKSEN, R. C. Evaluation of various spraying equipment for effective application of fungicides to control asian soybean rust. Aspects of Applied Biology 77, Warwick-UK, v. 1, p. 1-8, 2006.

PANNETON, B. Image analysis of water-sensitive cards for spray coverage experiments. ASABE - Applied Engineering in Agriculture, St. Joseph, MI, v. 18, n. 2, p. 179-182, 2002.

PARKIN, C. S. Methods for measuring spray droplet sizes. In: MATHEWS, G. A.; HISLOP, E. C. Application technology for crop protection. Wallingford, 1993, p. 5784.

PESSOA, M. C. P. Y.; CHAIM, A. Programa computacional para estimativa de uniformidade de gotas de herbicidas aplicados por pulverização aérea. Pesquisa Agropecuária Brasileira, Brasília, v. 34, n. 1, p. 45-56, 1999.

ROMAN, R. A. A.; CORTEZ, J. W.; FERREIRA, M. C.; OLIVEIRA, J. R. G. Cobertura da cultura da soja pela calda fungicida em função de pontas de pulverização e volumes de aplicação. Scientia Agraria, Curitiba, v. 10, n. 3, p. 223-232, 2009.

SALYANI, M.; FOX, R. D. Performance of image analysis for assesment of simulated spray droplet distribution. ASABE- Transaction of ASABE, St. Joseph, MI, v. 37, n. 4, p. 1083-1089, 1994.

SCHICK, R. J. An engineer's practical guide drop size. Wheaton: Spraying Systems, 1997. 28 p.

TURNER, C. R.; HUNTINGTON, K. A. The use of water sensitive dye for the detection and assessment of small spray droplets. Journal of Agricultural Engineering Research, v. 15, n. 4, p. 385-287, 1970.

VASQUEZ, J. Aplicación de productos fitosanitarios técnicas y equipos. Ediciones Agrotecnicas, 2004, 389 p.

VIANA, R. G.; FERREIRA, L. R.; TEIXEIRA, M. M.; CECON, P. R.; SOUZA, G. V. R. de Deposição de gotas no dossel da soja por diferentes pontas de pulverização hidráulica e pressões de trabalho. Engenharia na Agricultura, Viçosa, MG, v. 16, n. 4, p. 428-435, 2008.

ZAR, J. H. Biostatical analysis. 4. ed. Upper Saddle River, New Jersey: Prentice Hall, 1999. 663 p. 